\title{
Das Ende der Gleichheit? Der schwedische Wohlfahrtsstaat nach der Krise
}

Schweden leistet sich den größten Wohlfahrtsstaat der Welt und kann gleichzeitig durchgängig einen Überschuss in seinen öffentlichen Finanzen vorweisen. Darüber hinaus hat das schwedische Wohlfahrtsstaatsmodell während der letzten fünf Jahre unerwartete Unterstützung von der politischen Rechten bekommen. Dies spricht für eine hohe Belastbarkeit des Modells. Dennoch gibt es Zeichen wachsender Einkommensunterschiede, anhaltender ethnischer Segregation in Wohnvierteln und einer gewissen Marginalisierung von Gruppen der Gesellschaft - alleinstehende Mütter, Immigranten und Jugendliche. Diese Probleme deuten auf wachsende innere Widersprüche, die zukünftig zu Auflösungserscheinungen des schwedischen Wohlfahrtsstaats führen könnten.

\section{1 \\ Entstehung und gegen- wärtige Struktur des schwedischen Modells}

Auch wenn dieser Beitrag sich mit der $\mathrm{Zu}-$ kunft des schwedischen Modells auseinandersetzt, so ist es zunächst einmal wichtig, die geschichtliche Entwicklung des Systems zu rekapitulieren. Diese vorangegangenen Entscheidungen prägen die spezifische Ausbildung der sozialpolitischen Institutionen in Schweden, ihre Universalität ${ }^{1}$ und ihren Leistungsumfang. Als Weiterentwicklung der Armengesetze und der Unterstützungskassen führte Schweden zu Beginn des letzten Jahrhunderts Sozialversicherungen ein, die sich vom staatskorporatistischen Ansatz im bismarckschen Deutschland und anderen europäischen Ländern unterschieden. Das erste schwedische Rentengesetz von 1913 war sowohl beitragspflichtig als auch zielgruppenorientiert, das heißt es behielt die Bedürftigkeitsprüfung der Armengesetze bei, erweiterte aber die Anspruchsberechtigung, um einen Großteil der älteren Bevölkerung zu erfassen. Als die Bedürftigkeitsprüfung nach dem Zweiten Weltkrieg abgeschafft wurde, wurde - vergleichbar mit der britischen Beveridge Reform, aber ohne obligatorische Beiträge ein Grundsicherungsmodell mit einem einheitlichen Leistungssatz eingeführt. Zur gleichen Zeit wurde auch die Einführung eines allgemeinen Kindergeldes beschlossen. Mit dem Aufbau von verdienstabhängigen Leistungsansprüchen in den 1950er Jahren wurden die Programme zur Grundsicherung flächendeckend umgesetzt. Da- hinter stand die Idee, sowohl dem Bedürfnis nach Grundsicherung als auch nach Einkommenssicherheit $\mathrm{zu}$ entsprechen (Korpi/Palme 2003, 2004).

Darüber hinaus darf aber auch eine andere bedeutende Besonderheit des schwedischen Modells nicht vergessen werden: die Einführung der staatlichen Subventionierung von freiwilligen Versicherungsbeiträgen. In der Kranken- und Arbeitslosenversicherung führte der erste staatliche Eingriff dementsprechend zum Aufbau eines freiwilligen, staatlich subventionierten Modells. Im Jahr 1955 wurde die allgemeine schwedische Krankenversicherung flächendeckend eingeführt und man etablierte nach und nach das Prinzip verdienstabhängiger Leistungen. So wurde auch die Durchsetzung verdienstabhängiger Rentenzahlungen vorangetrieben. Am Ende des Jahrzehnts hatte Schweden so ein universalistisches Modell der sozialen Sicherung geschaffen. Trotz dieser Ausweitung verblieben weiterhin zielgruppenorientierte Elemente im System, wie beispielsweise das Wohngeld und das Sozialgeld, wogegen die Arbeitslosenversicherung in Schweden immer noch Elemente eines freiwilligen staatlich subventionierten Modells aufwies (Kangas/Palme 2005). In Bezug auf die Krankenversicherung wurden jedoch die letzten Spuren der Unterstützungskassen durch eine Verwaltungsreform im Jahr 2005 entfernt und die unabhängigen Versicherungsfonds in das staatliche Zentralsystem eingegliedert.

Das moderne schwedische Transferleistungssystem besteht folglich aus drei grundlegenden Bestandteilen: den sozialen Bürgerrechten einschließlich dem System der Alters- und Familienvorsorge, den für alle geltenden verdienstabhängigen Sozialversicherungsleistungen und den einkommensabhängigen Leistungen, wie dem Wohngeld für Familien mit Kindern und ältere Personen und dem Sozialgeld (Palme 2006).

Im Rahmen der Darstellung der Veränderungen während der Krise am Anfang der 1990er Jahre und danach wird dieser Artikel auch auf die Entwicklung der Einkommensungleichheit eingehen (Palme et al. 2002). Die schwedische Wohlfahrts-

\footnotetext{
1 Universalität verweist hier auf Wohlfahrtsstaaten, in denen ausgebaute soziale Ansprüche und Rechte für alle Bürgerinnen und Bürger, unabhängig von ihrem Sozial- und Versicherungsstatus oder ihrer Bedürftigkeit, dominieren. Universalistische Wohlfahrtsstaaten weisen typischerweise mehr Steuer- als Beitragsfinanzierung auf.
}

Joakim Palme ist Professor für Soziologie und Direktor des Institute for Future Studies, Stockholm. Arbeitsschwerpunkt: Vergleichende Wohlfahrtsstaatsforschung. e-mail: joakim.palme@framtidsstudier.se Johan Fritzell ist Professor für Soziologie und Forschungsdirektor am Institute for Future Studies, Stockholm. Arbeitsschwerpunkte: Vergleichende Studien zur Einkommensverteilung, Armut und Sozialpolitik. e-mail: johan.fritzell@framtidsstudier.se Åke Bergmark ist Professor für Soziale Arbeit und Forschungsdirektor am Institut for Future Studies, Stockholm. Arbeitsschwerpunkt: Sozialversicherungssysteme. e-mail: ake.bergmark@framtidsstudier.se

Übersetzung aus dem Englischen von Karin Vitols 


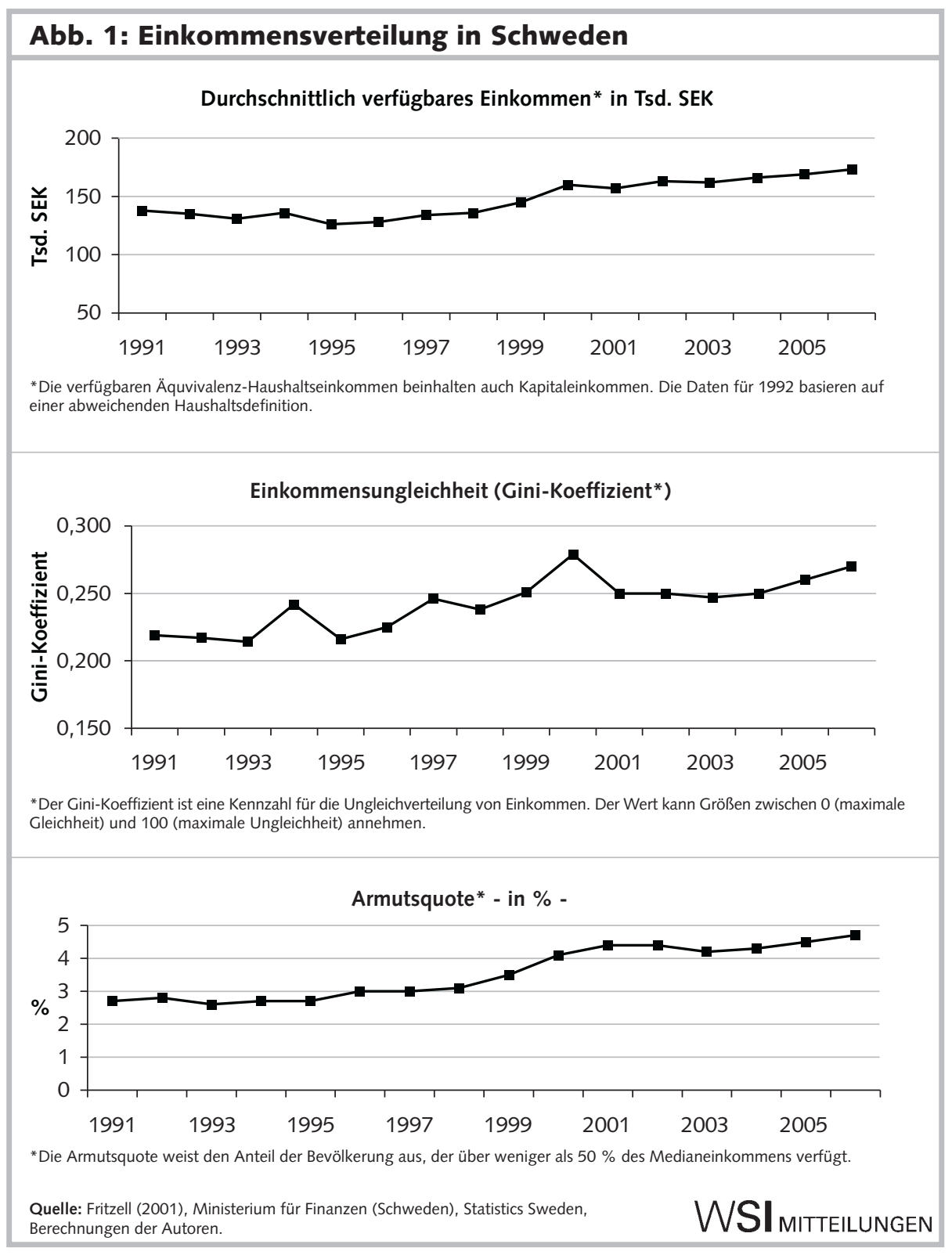

staatsforschung verfolgt hier einen mehrdimensionalen Ansatz, anstatt sich bei Untersuchungen nur auf das Einkommen oder die wirtschaftlichen Ressourcen zu beschränken. Studien über die Lebensbedingungen der Bevölkerung schließen so auch Bereiche wie Gesundheit, Ausbildung, Beschäftigung und politische Teilhabe ein. Da dieser Beitrag den Fokus auf die redistributiven Prinzipien legt, wird im Folgenden hauptsächlich der Bereich des Einkommens behandelt. Es gibt mehrere Gründe, warum der schwedische Fall insbesondere in Hinblick auf die Einkommensverteilung interessant erscheint. Eine Ursache ist, dass das Land relativ erfolgreich bei der Umsetzung seiner sozialpolitischen Ziele war und Armut und Ungleichheit reduzieren konnte. Ein weiterer Grund ist, dass Schweden
Im Folgenden wird die Entwicklung der durchschnittlichen Einkommen, der Ein- kommensungleichheit und der Armut seit 1991 dargestellt. Dieser Zeitraum schließt die große Wirtschaftskrise in den 1990er Jahren mit ein, als das Bruttoinlandsprodukt (BIP) in drei aufeinander folgenden Jahren schrumpfte und die offene Arbeitslosigkeit von weniger als $2 \%$ auf mehr als $8 \%$ anstieg. Darüber hinaus erhöhte sich in dieser Zeit auch der Anteil der Erwerbspersonen, die an Maßnahmen der aktiven Arbeitsmarktpolitik teilnahmen, von $2 \%$ auf fast $6 \%$. Diese Entwicklungen führten zu einer Krise in den öffentlichen Haushalten, die die damalige Regierung durch Steuererhöhungen und Leistungskürzungen, und damit zusätzlichem Druck auf die Einkommen der privaten Haushalte, zu bekämpfen versuchte (Palme et al. 2002; Palme 2006) .

Auffällig ist der Rückgang der Einkommen in der Beschäftigungskrise (Abbildung 1, oben). Die Werte stabilisierten sich erst gegen Ende der 1990er Jahre wieder und steigen seitdem an. Der überwiegende Anteil der Haushalte war zwar von dem Abschwung betroffen, konnte dann aber auch von dem Aufschwung profitieren. In Hinblick auf die Einkommensverteilung waren die unteren $90 \%$ vom Abschwung betroffen, während alle vom Wirtschaftsaufschwung profitieren konnten. Zwei treibende Kräfte standen hinter dem Anstieg des Durchschnittseinkommens: Das ist zum einen der Anstieg des Vermögens, der allerdings nur den oberen Teil der Einkommensverteilung erreichte und während des Beobachtungszeitraums großen jährlichen Schwankungen ausgesetzt war; zum anderen handelt es sich um die Erhöhung der Stundenlöhne, die allen Berufsgruppen zugute kam. Diese positive Entwicklung wurde jedoch durch nur bescheidene Verbesserungen in der Arbeitszeit gedämpft.

Die Entwicklung des Gini-Koeffizienten, der die Abweichung von der Gleichverteilung der Einkommen indiziert, zeigt für die Jahre 1991 bis 2006 (Abbildung 1, Mitte), dass die Einkommensungleichheit zugenommen hat. Sie unterliegt starken jährlichen Schwankungen. Spitzenwerte erreichte die Ungleichheit in den Jahren 1994 und 2000. In erster Linie sind diese Schwankungen auf Veränderungen im Steuersystem zurückzuführen, die den Zeitpunkt beeinflussten, zu dem Haushalte ihre Vermögensgewinne realisieren konnten. Die Einkommensungleichheit, gemessen am Gini-Koeffizienten, hat ihren Höchststand im Jahr 2000 erreicht, als der Koeffi- 
zient 0,279 betrug. Diesem Höchststand folgte ein Rückgang auf rund 0,250 und eine weitere Zunahme zwischen 2004 und 2006, die in erster Linie (aber nicht ausschließlich) von der gestiegenen Bedeutung der Vermögensgewinne und ihrer ungleichen Verteilung verursacht wurde. Im internationalen Vergleich ist die Einkommensungleichheit in Schweden allerdings nach wie vor niedrig (Atkinson 2003).

Einen weiteren Aspekt der Einkommensungleichheit stellt die Armutsquote dar (Abbildung 1, unten). Wie viele vergleichende Studien bezieht sich auch dieser Beitrag auf den Standardindikator des Bevölkerungsanteils mit einem Einkommen von unter $50 \%$ des Medianlohns. Dieser zeigt nur geringe Veränderungen im Laufe der Zeit, was angesichts der schwerwiegenden Wirtschaftskrise und der hohen Arbeitslosigkeit erstaunlich ist. Im internationalen Vergleich befinden sich die Armutsquoten in Schweden und in den anderen nordischen Ländern weiterhin auf niedrigem Niveau (Atkinson 2003).

Neben der Untersuchung dieser allgemeinen Trends ist es auch wichtig, bestimmte benachteiligte Gruppen auszumachen und die Entwicklung ihrer sozioökonomischen Lage zu verfolgen. In einer Analyse der 1990er Jahre konnte die Schwedische Wohlfahrtskommission (Swedish Welfare Commission) aufzeigen, dass die meisten soziodemografischen Gruppen von der Rezession und ihren Nachwirkungen betroffen waren (Palme et al. 2002). Allerdings waren die Auswirkungen bei drei Gruppen besonders groß: Jugendliche, Migranten und alleinerziehende Mütter (Bergmark/Palme 2003). Nach der Wirtschaftskrise hat sich auch für diese drei Gruppen das durchschnittliche Einkommen verbessert. Bei den jungen Erwachsenen und Einwanderern fiel der durchschnittliche Anstieg bei Frauen höher aus als bei Männern. Die Verbesserungen für alleinerziehende Mütter waren dagegen recht bescheiden. In Hinblick auf die soziale Ausgrenzung stellt das gestiegene Einkommen der Zuwanderer eine bemerkenswerte Entwicklung dar und bietet Anlass zur Hoffnung auf eine bessere Arbeitsmarktintegration dieser Beschäftigungsgruppe, vor allem, weil die Armut in Schweden primär mit fehlenden Einkommen aus Erwerbstätigkeit zu tun hat. Umfrageergebnisse über wirtschaftliche Notlagen weisen ebenfalls auf Verbesserungen für benachteiligte Gruppen im neuen Jahr- hundert hin. Mehr Menschen besitzen beispielsweise eine sogenannte Barmarge. ${ }^{2}$ Eine solche finanzielle Flexibilitätsreserve fehlt vielen Gruppen, so beispielsweise alleinerziehenden Müttern, und sie nahm während der ersten fünf Jahre dieses Jahrhunderts von $50 \%$ auf $41 \%$ ab.

\section{Neuer Trend zur Polarisierung}

In einer weiteren Untersuchung über die Veränderung der Lebensbedingungen stellen Bergmark/Fritzell (2007) auch fest, dass auf die Frage, wer vom System „aufgefangen " und wer ,fallengelassen "wurde, keine klare Antwort gegeben werden kann. Umfassende Analysen deuten darauf hin, dass eine Polarisierungstendenz unter gleichzeitiger Berücksichtigung verschiedener Dimensionen der Wohlfahrt insbesondere bei Jugendlichen, Migranten und alleinerziehenden Müttern zu beobachten ist. In wirtschaftlich guten Zeiten berichtete ein höherer Anteil der benachteiligten Gruppen, dass sie erwerbstätig seien, ihre Gesundheit gut sei und sie im Besitz einer Barmarge seien. Allerdings konnte kein entsprechender Rückgang des Anteils der Personen, die von schwierigen Lebensbedingungen in Bezug auf diese drei Wohlfahrtsdimensionen (fehlende Beschäftigung, Erkrankungen und wenig finanzielle Ressourcen) berichteten, verzeichnet werden. Die letzten beiden Dimensionen hatten zum Beispiel unter den alleinerziehenden Müttern in den Jahren 2004 und 2005 doppelt so hohe Werte wie in den späten 1990er Jahren. Solch eine Polarisierung der materiellen Lage kann auch bei den Sozialhilfeempfängern festgestellt werden. Sowohl die Kosten für Sozialhilfe als auch die Gesamtzahl der Empfänger sanken in den letzten zehn Jahren. Wenn jedoch die Wahrscheinlichkeit des Ausscheidens aus der Sozialhilfe berechnet wird, zeigt sich, dass diese heute niedriger ist als je zuvor. Die durchschnittliche Verweildauer in Sozialhilfe, die lange Zeit ca. vier Monate pro Jahr betrug, hat erheblich zugenommen und stieg im Jahr 2006 auf ein Rekordniveau von nahezu sechs Monaten an. Auch bei neueren Einwanderern deuten einige Beispiele auf eine Polarisierung hin. Frühere Studien haben gezeigt, dass im Vergleich zu anderen Migranten Personen aus dem ehemaligen Ju- goslawien äußerst erfolgreich bei einer raschen Eingliederung in den regulären Arbeitsmarkt waren. Dennoch zeigen langfristige Zeitreihen zur Sozialhilfe, dass zur gleichen Zeit eine recht große Anzahl von Ex-Jugoslawen längere Zeit in der Sozialhilfe verweilte. Ebenfalls lässt sich eine beunruhigende Tendenz bei der räumlichen Segregation von Personengruppen feststellen. Die sozioökonomische und ethnische Segregation in Bezug auf die Wohngebiete hat zugenommen. Diese Segregation ist der räumliche Ausdruck der sozialen Ungleichheiten. In allen Gesellschaften finden soziale und wirtschaftliche Ungleichheiten ihre Entsprechungen in der Wohnsituation. Allerdings sind Segregationsprozesse politisch beeinflussbar und damit veränderbar. Die bisherige Wohnungs- und Städtebaupolitik versuchte, so auch größtenteils der Segregation entgegenzuwirken.

\section{Eine neue Politik der Umverteilung}

Viele Forschungsergebnisse weisen darauf hin, dass die soziale Ungleichheit größtenteils auf individuelle Eigenschaften zurückzuführen ist (Bermark/Fritzell 2007). Warum sollte man also über eine zunehmende Segregation besorgt sein? Es gibt mehrere Gründe, warum eine Segregation als Alarmzeichen wahrgenommen werden muss: Der wichtigste Grund ist, dass das Prinzip universeller Teilhabe durch geografisch getrennte Wohnlagen von Arm und Reich sowie Migranten und Einheimischen bedroht wird. Segregation ist ein guter Nährboden für Vorurteile und selbstgefällige Einschätzungen. In einer solchen Gesellschaft würde es also immer schwieriger werden, die Unterstützung für einen universalistischen Wohlfahrtsstaat zu erhalten. Eine weitere Herausforderung stellt die hohe Armutsquote unter Einwanderern und jungen Personen ohne Kinder dar. Eine neue Aufgabe für Schweden ist es deshalb,

\footnotetext{
2 Die Barmarge ist ein vom schwedischen Statistikamt festgelegter Betrag, der die finanzielle Flexibilität misst. Wer in der Lage ist, innerhalb einer Woche 14.000 schwedische Kronen (ca. 1.500 Euro) in bar zu besorgen, gilt als finanziell flexibel. Dies wird als Indikator im jährlich erscheinenden 'Swedisch Survey of Living Conditions' erhoben.
} 
erfolgreiche Strategien zur Bekämpfung dieser neuen Armut zu entwickeln.

Im Hinblick auf die Einkommensumverteilungspolitik lassen sich für die 1990er Jahre drei Phasen abgrenzen: die große Steuerreform, das Krisenmanagement und die wirtschaftliche Erholung (Palme et al. 2002). Die erste Phase führte durch weniger progressiv gestaffelte Steuersätze und großzügigere Sozialleistungen zu einer Verschiebung der Ausgaben vom Steuersystem zum Transfersystem. Das Ausmaß der Verteilungseffekte blieb allerdings weitgehend unverändert. Die zweite Phase beinhaltete sowohl Leistungskürzungen als auch Steuererhöhungen. Da der Umfang der Kürzungen aber den der Erhöhungen leicht übertraf, nahm die Umverteilung in dieser Zeit etwas ab. Die dritte Phase ist von Steuersenkungen und Leistungserhöhungen geprägt, die unter Verteilungsgesichtspunkten nur zu kleinen Änderungen geführt haben.

Im ersten Jahrzehnt des 21. Jahrhunderts können zwei Phasen abgegrenzt werden: Die erste stellt eine Fortsetzung der Trends dar, die sich bereits in den 1990er Jahren im Aufschwung abzeichneten. Die zweite Phase ist von politischen Maßnahmen geprägt, die nach dem Regierungswechsel von einer sozialdemokratischen Minderheitsregierung zu einer Mitte-RechtsMehrheitskoalition umgesetzt wurden.

Die erste Phase führte zu Steuererhöhungen sowie einer schrittweisen Verbesserung der Arbeitslosenversicherung und einer Erhöhung des Kindergeldes. Außerdem wurde im Jahr 2003 das Rentensystem reformiert (Palme 2003). Trotz der Reform wurde garantiert, dass kein Rentner eine geringere Rentenleistung oder ein geringeres verfügbares Einkommen im ersten Jahr des Ruhestandes erhalten sollte als nach dem alten System. Im Jahr 2006 wurde die Beitragsbemessungsgrenze beim Krankengeld angehoben, was dazu führte, dass Personen mit mittleren bis hohen Einkommen besser im gesetzlichen Versicherungssystem abgesichert wurden. Darüber hinaus hat die Regierung auch die öffentlichen Ausgaben für Familien mit Kindern erhöht, und zwar in Form einer Anpassung der Kindergeldzahlungen für Studierende, einer Erhöhung des Wohngelds, höherer Vorauszahlung von Unterhaltsleistungen an alleinerziehende Eltern und der Einführung von Leistungsansprüchen bei der Geburt eines zweiten Kindes.

In der zweiten Phase kam es allerdings unter der Mitte-Rechts-Koalition zu einem politischen Richtungswechsel. In diesem Zeitraum wurden vielfältige Leistungskürzungen und Steuererleichterungen eingeführt. Das soziale Sicherungssystem verzeichnete sowohl eine Reduzierung der Beitragsbemessungsgrenzen als auch eine Kürzung der Lohnersatzleistungen, beispielsweise für Langzeitarbeitslose. Diese Veränderungen waren eher unerheblich, obwohl sie zu einem etwas höheren Maß an Ungleichheit beitrugen. Die Steuererleichterungen beinhalteten mehr Abschreibungsmöglichkeiten für das Erwerbseinkommen, die Abschaffung der Vermögenssteuer und eine weniger progressiv gestaffelte Eigentumssteuer.

Alles in allem deuten diese Entwicklungen im Umverteilungssystem auf eine Nutzenverlagerung zulasten der Arbeitslosen und zugunsten der Beschäftigten hin. Die Regierung hatte sich explizit zum Ziel gesetzt, die Zahl der Arbeitslosen durch eine Stärkung der Arbeitsanreize zu reduzieren. Für das universale Modell der sozialen Sicherung ergeben sich hieraus Probleme, weil entsprechende Voraussetzungen nicht mehr erfüllt werden. Generell hat Schweden durch das Prinzip der Preisindexierung im Sozialversicherungssystem Schwierigkeiten, die Einkommensbemessungsgrenzen bei der Sozialversicherung an das Wachstum der Reallöhne anzupassen. Dies könnte wiederum auf lange Sicht dazu führen, dass die Unterstützung der Mittelschicht für den Sozialstaat zurückgeht (Palme 2006).

Die schwedischen Rentenreformen von 1994 und 1998 stellten einen Neuanfang dar und sind für die Interpretation der gegenwärtigen Situation, aber auch in weiteren Kontexten von besonderer Bedeutung (Palme 2003). Durch die Indexierung der Einkommensgrenzen für Leistungszwecke an die Reallöhne stärkte diese Reform in mehrfacher Hinsicht das universale Sicherungsmodell. Dies bedeutet, dass etwa derselbe Anteil der Arbeitskräfte auch weiterhin einen Einkommensersatz aus dem gesetzlichen System erhalten wird. In dem alten preisindexierten System erreichte dagegen eine zunehmende Zahl von Personen ein Einkommen, das durch reale Lohnerhöhungen oberhalb der Einkommensbemessungsgrenze lag.

Auch andere Aspekte der Rentenreform haben möglicherweise erhebliche Auswirkungen auf die Zukunft. Das gilt für den vollfinanzierten Teil des reformierten Systems, der zwar individuell, aber obligato- risch ausgestaltet ist. Hier könnte argumentiert werden, dass die individuelle Risikofreude dem Sozialversicherungssystem entgegensteht, sie aber zur gleichen Zeit auch eine individuelle Wahl ermöglicht. Die vergleichsweise hohen Verwaltungskosten des Systems führen zudem dazu, dass die Renten niedriger ausfallen werden als bisher üblich.

Ein weiteres Beispiel stellt die neue Methode der Koordination der Grundsicherung mit anderen Leistungen dar: Früher erhielten alle Rentner einen Teil ihrer Rente in Form einer gemeinsamen Pauschale. Das reformierte System baut hingegen hauptsächlich auf die einkommensbasierte Rente. Leistungen der Grundsicherung werden nur noch denjenigen gewährt, die nicht genug verdient haben, um das Mindestniveau zu übersteigen. Diese neue Strategie beinhaltet, um den Universalismus des Systems aufrechtzuerhalten, auch Maßnahmen zur Förderung der Arbeitsaufnahme für Personen mit niedrigem Einkommen. Bisher zeichnen sich die politischen Konsequenzen noch nicht $\mathrm{ab}$, die zukünftig aus dieser Grundsicherung in einem System, das hauptsächlich auf die einkommensabhängige Rente setzt, resultieren werden.

Mit Blick auf Umverteilungsstrategien spielen die Sozialfürsorge und die in ihr stattfindenden Veränderungen eine wichtige Rolle. Eine Analyse dieses Bereiches zeigt, wie sich Schweden mit dem Dilemma der Einbindung der Mittelschicht in einem umverteilenden sozialpolitischen System auseinandergesetzt hat. Historisch gesehen weicht die Entwicklung der Wohlfahrtspflege, einschließlich der Gesundheitsfürsorge, in vielerlei Hinsicht von den obigen Darstellungen ab. Und das nicht nur, weil die Fürsorgeleistungen von den Geldleistungen sowohl administrativ als auch finanziell getrennt worden sind, sondern auch, weil die für sie ausgegebenen Beträge Schweden in internationalen Vergleichen $\mathrm{zu}$ einer Ausnahmeerscheinung machen (Korpi/Palme 2004).

Die Universalität der Ansprüche auf Sozialleistungen in Schweden ist im Großen und Ganzen ein Nachkriegsphänomen. Schweden führte Mitte der 1950er Jahre ein universales Gesundheitsfürsorgesystem ein, das später durch den Aufbau der Altenhilfe und Jugendfürsorge noch ergänzt wurde. Nach und nach ist auch die soziale Absicherung für arbeitsunfähige Personen Teil des universalen Sicherungssystems geworden. In diesem Zusammen- 
hang scheint es auch wichtig, auf die Bedeutung von Vollbeschäftigung, aktiver Arbeitsmarktpolitik und Doppelverdienersystem hinzuweisen, die das Modell ferner mitgeprägt haben (Kangas/Palme 2005).

Die Ausweitung der sozialen Ansprüche führte zu steigenden Ausgaben. In Schweden kam verschärfend hinzu, dass das Land die weltweit älteste Bevölkerung aufweist. Allerdings weichen die Nettokosten des schwedischen Transferleistungssystems nicht stark von denen anderer westeuropäischer Modelle ab. Das liegt an mehreren Faktoren, wobei die wichtigsten Gründe das relativ hohe formale und tatsächliche Rentenalter sowie die hohe Erwerbsbeteiligung der Frauen sind (Kangas/ Palme 2005).

Von der schwedischen Tradition starker Steuerfinanzierung universaler Leistungen, ergänzt durch Subventionen für freiwillige Versicherungen, gibt es jedoch diverse Abweichungen:

(1) Die Gemeinden besaßen immer eine starke finanzielle Verantwortung im Bereich der Sozialhilfe und insbesondere hinsichtlich der Betreuung von „gefährdeten“ Personengruppen.

(2) Die Einführung von einkommensabhängigen Leistungen wurde an neue Finanzierungsmodelle gekoppelt, wobei die Sozialversicherungsbeiträge von den Arbeitgebern finanziert wurden. Diese Sozialversicherungsbeiträge wurden auch zur Finanzierung der Arbeitsmarktpolitik und der Kindertagesstätten verwendet.

(3) Eines der Merkmale der jüngsten Rentenreform ist nun, dass die Beiträge der Versicherten auch tatsächlich dem Rentensystem zufließen. Diese „Benutzerfinanzierung" in fast allen Bereichen der Fürsorge ist für das schwedische soziale Sicherungsmodell nichts Neues.

Solche Abweichungen relativieren den universalistischen und umverteilenden Anspruch des Modells. So hat die Erhöhung der Beiträge in der Krise der 1990er Jahre bei ökonomisch benachteiligten Gruppen überdurchschnittlich dazu geführt, auf die Gesundheits- und Altersvorsorge zu verzichten (eingeschränkte Universalität). Gleichzeitig tragen sie aber durch ihre Steuerzahlungen weiterhin zur Finanzierung solcher Dienstleistungen bei (negativer Umverteilungseffekt).

\section{Reform der sozialen Dienstleistungen}

Die Veränderungen bei der Finanzierung der Sozialleistungen, die in den ersten Jahren des 21. Jahrhunderts stattfanden, veranschaulichen gut, wie Schweden mit dem Dilemma der Einbindung der Mittelschicht in das umverteilende soziale Sicherungssystem umging. Dieses Dilemma besteht darin, dass Umverteilungspolitiken schwerlich ohne die prinzipielle Unterstützung der Mittelschichten durchgesetzt und aufrechterhalten werden können; Mittelschichten sind jedoch häufig von progressiv gestalteten Steuern und Abgaben belastet, profitieren aber nicht in gleichem $\mathrm{Maß}$ von stark umverteilenden Aspekten des Wohlfahrtsstaats. Vor diesem Hintergrund wurden vor allem Bemessungshöchstgrenzen für Kindergartengebühren sowie für Dienstleistungen in der Altenpflege eingeführt (Palme 2006).

Der Zweck der Einführung von Höchstbeträgen für die Kinderbetreuungskosten bestand darin, der Situation von Familien mit niedrigem Einkommen gerecht zu werden, da die Gebührenhöhe sie ansonsten davon abhalten könnte, diese stark subventionierten aber ggf. dennoch kostspieligen Serviceangebote in Anspruch zu nehmen. Ein weiteres Problem wurde in den zunehmenden Randerscheinungen (Armutsfallen) gesehen, die insbesondere Ein-ElternFamilien durch das Zusammentreffen der einkommensabhängigen Gebühren für die Kinderbetreuung und der einkommensabhängigen Gewährung von Wohngeld betrafen. Die Verschlechterung der wirtschaftlichen Situation der Ein-Eltern-Familien in den 1990er Jahren ist Folge der niedrigeren Einkünfte aus nicht selbstständiger Arbeit (und nicht Folge niedriger Leistungen).

In der Altenpflege lagen die Motive etwas anders. Die Sorge war hier teilweise, dass die finanzielle Belastung für die Angehörigen der in Heimen lebenden älteren Menschen durch sehr hohe Nutzungsentgelte untragbar werden könnte. Außerdem hat die Gefahr hoher Kosten auch zu Vermeidungsstrategien bei dem Teil der Haushalte geführt, der von hohen Gebühren betroffen ist (Überschreibung von Vermögen auf Verwandte).

Bedeutsam sind außerdem mehrere Veränderungen in der Organisation der so- zialen Dienstleistungen. Hierzu gehört die Einführung von Käufer-Anbieter-Modellen (purchaser-provider models), die, zusammen mit einer Gesetzesänderung im Jahr 1992, kommerzielle Anbieter in allen Bereichen zuließ und zu einer Neugestaltung der sozialen Dienste führte (Palme et al. 2002). Zwar entscheiden die Gemeinden und Landkreise weiterhin über die Auftragsvergabe bei sozialen Dienstleistungen, aber die Reform hat dazu geführt, dass sich der Anteil der privaten Bereitstellung öffentlich finanzierter Dienstleistungen deutlich erhöhte. Es gibt allerdings große Unterschiede zwischen Branchen und Regionen. Die privaten Versorger haben auch nicht in allen Fällen eine freie Kundenwahl (customer-choice-modelle), sondern erhalten ihre Aufträge im Rahmen von Ausschreibungsverfahren. Die öffentlichen Monopole bestehen also weiterhin fort, aber einige Aufgaben werden an private Dienstleister abgegeben.

Die Wahlfreiheit kann jedoch sehr wohl als Wert an sich angesehen werden. Eine entscheidende Frage für das schwedische Modell ist nun, ob es gelingen kann, die vorhandenen Angebote mit den an sie gestellten Anforderungen in Einklang zu bringen. Die Wahlfreiheit im öffentlich finanzierten sozialen Dienstleistungssektor führte als Folge der Einführung von Customer-Choice-Modellen in einigen Fällen zu einer zunehmenden Segregation der Nutzerkreise. So könnte der Wert des Bildungssystems - als Schnittstelle für verschiedene Gruppen in der Gesellschaft im Widerspruch zu den Zielen der Wahlfreiheit und anderen Vorteilen stehen, die sich aus einem verstärkten Wettbewerb ergeben sollen. Die Abwägung verschiedener Ziele sollte deshalb im Idealfall auf entsprechenden Expertenbewertungen und einer genauen Analyse, wie die verschiedenen Alternativen sich in der Praxis umsetzen lassen, basieren.

\section{Fazit}

Die verschiedenen politischen Veränderungen, die seit 1990 stattgefunden haben, führten bisher nicht zu einem grundlegenden Pfadwechsel in der schwedischen Umverteilungsstrategie. Gegenläufige Tendenzen des Wandels existieren nebeneinander. Einige Änderungen zielen darauf ab, die 
etablierte Umverteilungsstrategie zu unterstützen. Andere beschriebene Veränderungen könnten zu einer grundlegenden Interessenverschiebung im Hinblick auf den Erhalt des Wohlfahrtsstaats führen. Zum Beispiel wurden Schwierigkeiten bei der Nutzung des Steuersystems zu Umverteilungszwecken aufgezeigt: Steuersenkungen, die in einem Zusammenhang mit hohen Grenzsteuersätzen stehen, führten in der Vergangenheit dazu, dass Umverteilungsziele aus dem Steuersystem zu den Transferleistungen verlagert wurden (Palme et al. 2002).
Die Finanzierung des Wohlfahrtsstaates wird entscheidend für seine Zukunft sein. Eine aktuelle Herausforderung sind die hohen Kostenanforderungen, die die Bedürfnisse einer älter werdenden Gesellschaft an die öffentliche Hand und die verschiedenen Quellen der Finanzierung stellen. Ein weiteres Problem ist, dass die Kritik am Sozialstaat sich in der Regel auf Behauptungen wie mangelnde Kostenkontrolle und zu wenig Arbeitsanreize stützt. Der Anteil der älteren Bevölkerungsgruppen nimmt auf lange Sicht immer mehr zu, während die absoluten Zahlen der anderen
Altersgruppen abnehmen. In den Jahren 2025-2030 wird dieser Alterungsprozesses sogar noch dramatischer ausfallen und nur die älteste Bevölkerungsgruppe mit einem Alter von 80 Jahren oder älter wird überhaupt noch einen Anstieg verzeichnen.

In naher Zukunft stellt die Alterung der Bevölkerung in Schweden noch kein Problem für das Umverteilungssystem dar. Es ist jedoch an der Zeit, sich Gedanken zu machen, wie das schwedische Modell der Zukunft aussehen soll, um dann das Steueraufkommen entsprechend hieran auszurichten!

\section{LITERATUR}

Atkinson, A. B. (2003): Income Inequality in OECD Countries: Data and Explanations, CESifo Working Paper 881

Bergmark, Å./Fritzell J. (Hrsg.) (2007): Välfärdens ansikte mot 2000-

talet, Welfare facing the 21 century, Socialvetenskaplig tidskrift (special issue), S. 2-3

Bergmark, Å./Palme, J. (2003): Welfare and the Unemployment Crisis: Sweden in the 1990s, in: International Journal of Social Welfare, S. 108122

Kangas, O./Palme J. (Hrsg.) (2005): Social Policy and Economic Development in the Nordic Countries, Basingstoke

Korpi, W./Palme, J. (2003): Klassenpolitik und Wohlfahrtsstaatsabbau: Kürzungen von Rechten der sozialen Sicherung in 18 Ländern 19751995, in: Müller, W./Scherer, St. (Hrsg.): Mehr Risiken - Mehr Ungleichheit? Abbau des Wohlfahrsstaates, Flexibilisierung des Arbeitsmarktes und die Folgen, Frankfurt, S. 221-255
Korpi, W./Palme, J. (2004): Robin Hood, St. Matthew, or simple egalitarianism? Strategies of equality in welfare States, in: Kennet, P.: A Handbook of Comparative Social Policy, Cheltenham, S. 153-179

Palme, J. (2003): Pension reform in Sweden and the changing boundaries between public and private, in: Clark G. L./Whiteseide, C.: Pension Security in the $21^{\text {st }}$ Century, Oxford, S. 144-167

Palme, J. (2006): Income distribution in Sweden, in: The Japanese Journal of Social Security Policy 1, S. 16-26

Palme, J./Bergmark, Å./Bäckman, O./Estrada, F./Fritzell, J./Lundberg, O./Sjöberg, O./Szebehely, M. (2002): WelfareTrends in Sweden: Balancing the Books for the 1990s, in: Journal of European Social Policy 4, S. 329-346 